Vingt-deux ans après

la découverte de la supra-

conductivité dans des oxydes

de cuivre, une deuxième

famille de matériaux supra-

conducteurs à haute tempéra-

ture critique, cette fois-ci

à base de fer, a été mise

au jour. Plus qu'une heureuse

surprise, cette nouvelle

découverte prouve qu'il y a

différentes voies pour

atteindre des températures

critiques élevées.

Les applications de ces pnic-

tures et chalcogénures de fer

demeurent pour l'instant

confidentielles. Mais l'origine

de leur supraconductivité,

potentiellement différente

de celle des cuprates, souligne

la grande fécondité de

la physique des solides,

et constitue un nouveau défi

de taille pour la théorie.

(1) Pnictures : composés contenant des éléments de la colonne de l'azote du tableau périodique : $\mathrm{N}, \mathrm{P}, \mathrm{As}, \mathrm{Sb}$

(2) Magnétisme itinérant : magnétisme qui apparait dans des matériaux métalliques, dans lesquels les électrons sont assez fortement délocalisés sans être totalement libres ; c'est le cas du magnétisme du fer, du cobalt et du nickel.

\title{
Les supraconducteurs à base de fer
}

Philippe Bourges (philippe.bourges@cea.fr) et Yvan Sidis (yvan.sidis@cea.fr) Laboratoire Léon Brillouin, UMR 12 CEA-CNRS, CEA Saclay, 91191 Gif-sur-Yvette Cedex

À la grande surprise de la communauté scientifique, une supraconductivité à haute température critique $\left(T_{\mathrm{c}}>50 \mathrm{~K}\right)$ a été découverte dans des composés à base de fer, en mars 2008 [1]. En effet, le fer, élément magnétique, était plutôt considéré comme antagoniste de la supraconductivité. De plus, c'était la première fois qu'on atteignait une température critique si élevée sans cuivre ni oxygène. De même que les oxydes de cuivre supraconducteurs, ces nouveaux composés présentent une structure lamellaire avec, cette fois, des couches de pnicture ${ }^{(1)}$ de fer (Fe et As ou $\mathrm{P}$ ) ou de chalcogénure de fer ( $\mathrm{Fe}$ et $\mathrm{Se}$ ou $\mathrm{Te}$ ), entre lesquelles s'intercalent également des plans " réservoirs de charges " (voir l'image de couverture). Ces matériaux présentent un diagramme de phase proche de ceux des cuprates avec, en fonction du dopage, une phase antiferromagnétique, puis la phase supraconductrice (fig. 1).
Cependant, il est apparu rapidement que les propriétés des cuprates et des supraconducteurs à base de fer sont assez différentes. En particulier, alors que dans le cas des cuprates, le composé parent non dopé est un isolant avec un magnétisme localisé, les pnictures $^{(1)}$ de fer restent métalliques à dopage nul, avec un magnétisme itinérant ${ }^{(2)}$ induit par des propriétés d' "emboîtement " de la surface de Fermi. Les interactions électroniques semblent de fait plus faibles que dans les cuprates. Les cinq orbitales $d$ du fer apportent les électrons de conduction, et sont potentiellement toutes impliquées dans la supraconductivité, ce qui induit un degré de complexité supplémentaire dans ces systèmes. Les mesures de photoémission résolue en angle et les calculs de bandes électroniques s'accordent sur l'existence de plusieurs surfaces de Fermi de trous et d'électrons, sur lesquelles différents gaps

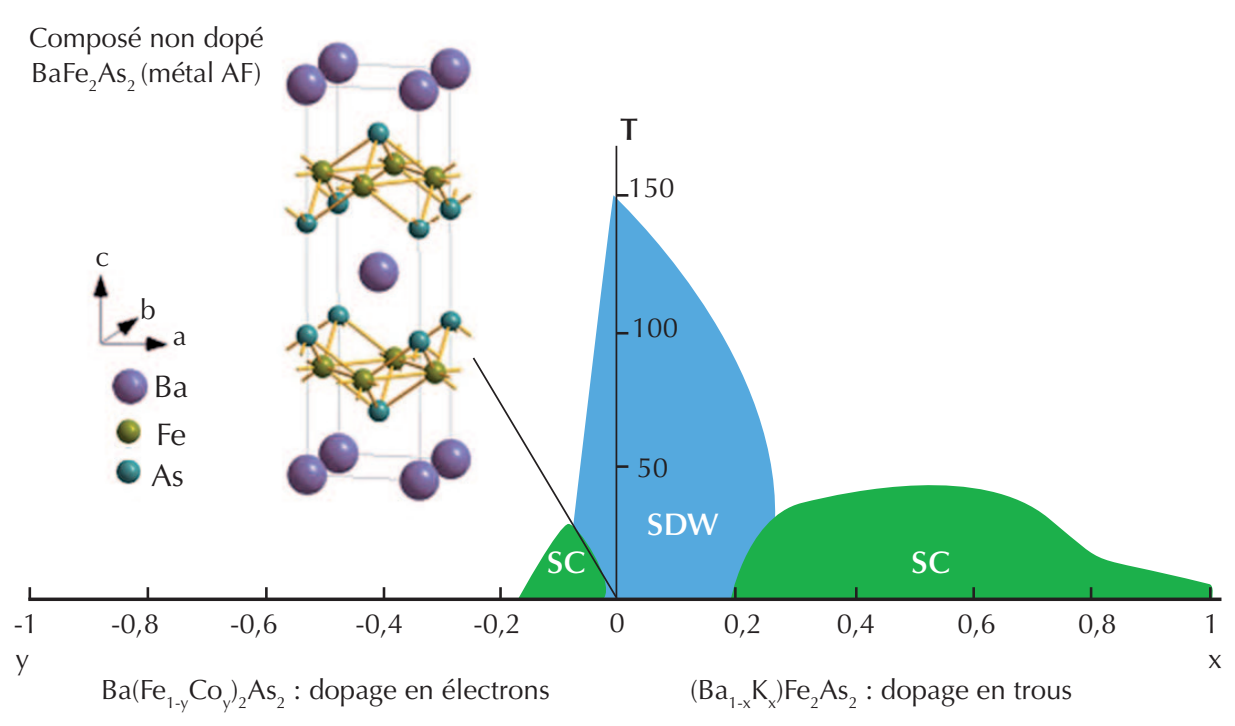

1. Diagramme de phase du pnicture de fer $\mathrm{BaFe}_{2} \mathrm{As}_{2}$, en fonction d'un dopage en trous $(\mathrm{x})$ ou en électrons (y), avec une phase supraconductrice (SC) de chaque côté. Le dopage en électrons est obtenu en substituant des atomes de cobalt aux atomes de fer, le dopage en trous en substituant du potassium au baryum. À dopage nul, à la différence des cuprates, le système reste métallique, avec un ordre antiferromagnétique caractérisé par une onde de densité de spins (SDW). La structure cristalline du composé non dopé BaFe $2 \mathrm{As}_{2}$ comporte des blocs d'atomes de fer (jaunes) et d'arsenic (turquoise), séparés par des plans de baryum (atomes violets). 
Spectromètre trois-axes 2T du Laboratoire Léon Brillouin (CEA-CNRS, Saclay) pour l'étude par diffusion inélastique de neutrons thermiques (DIN, voir encadré p. 9) des excitations cristallines et magnétiques dans des matériaux monocristallins. De droite à gauche protection biologique du monochromateur (en orange), l'axe échantillon (avec le cryostat rouge), l'axe du cristal analyseur, et la protection du détecteur (en blanc).

supraconducteurs se développent. Les gaps, dits de symétrie $s_{ \pm}$, sont isotropes avec un changement de signe entre les différents feuillets de la surface de Fermi. Plusieurs mesures récentes indiquent cependant l'existence de " nœuds " au niveau de Fermi (c'est-à-dire de points de la surface de Fermi où le gap est nul) [2].

La plupart des théories de la supraconductivité sont basées sur l'existence d'un boson, déjà présent dans l'état normal, médiateur de l'attraction entre électrons. Dans le cas des pnictures de fer, les vibrations du réseau ne sont pas de bons candidats, car l'interaction électron-phonon est faible dans ces matériaux. Le diagramme de phase et la symétrie non conventionnelle du paramètre d'ordre suggèrent un appariement des paires de Cooper via un mécanisme d'origine purement électronique, mettant en jeu les fluctuations magnétiques.

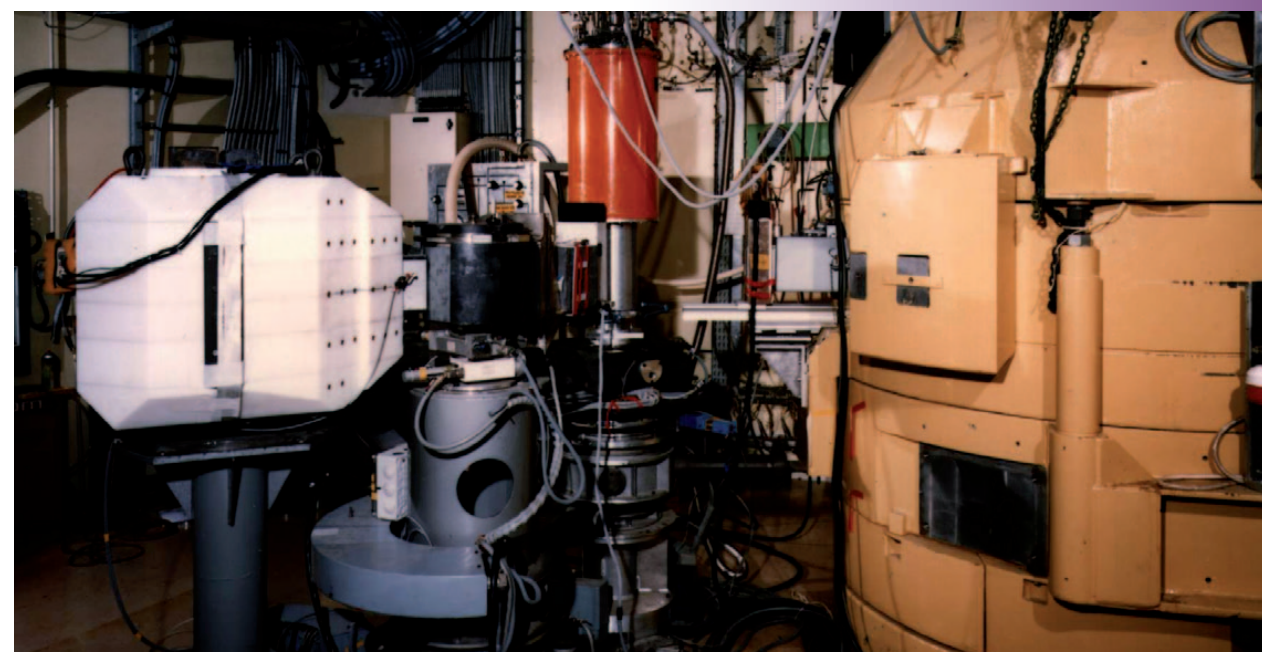

Les électrons s'apparieraient en utilisant les fluctuations qu'ils ont eux-mêmes engendrées sous l'effet de leurs interactions. Les propriétés des électrons, des paires de Cooper et des fluctuations magnétiques sont alors toutes reliées les unes aux autres, et doivent être traitées de façon autocohérente. Pour être confortée, cette approche requiert une description quantitative du spectre des fluctuations magnétiques, ainsi qu'une estimation de l'amplitude du couplage $V$ entre les électrons et ces fluctuations. Là encore, la diffusion inélastique de neutrons (voir encadré, p. 9) a été utilisée [3] pour mettre en évidence de fortes fluctuations magnétiques dans la phase normale autour du dopage optimal. En passant dans l'état supraconducteur, un pic de résonance apparaît dans le spectre des fluctuations magnétiques, comme attendu pour une symétrie $s_{ \pm}$ du paramètre d'ordre supraconducteur.
L'énergie de cette excitation résonnante diminue avec la température, suivant exactement l'évolution thermique de l'énergie nécessaire pour briser une paire de Cooper, comme le prédit la théorie.

Si la découverte de la supraconductivité des matériaux à base de fer est une excellente nouvelle pour la physique des solides, leur développement pour les applications pose de nombreux problèmes. Leur supraconductivité, de type $s$, les rend moins vulnérables à la présence de défauts cristallins que les cuprates. Cependant, la fabrication d'échantillons suffisamment purs et de grande taille n'en est encore qu'à ses balbutiements.

\section{Références}

1• Y. Kamihara et al., J. Am. Chem. Soc. 130 (2008) 3296

2• P.J. Hirschfeld et al., Rep. Prog. Phys. 74 (2011) 124508

3• D.S. Isonov et al., Nature Phys. 6 (2010) 178. 